\title{
Choice of Contact Points during Multidigit Grasping: Effect of Predictability of Object Center of Mass Location
}

\author{
Jamie Lukos, ${ }^{1}$ Caterina Ansuini, ${ }^{3}$ and Marco Santello ${ }^{1,2}$ \\ ${ }^{1}$ Department of Kinesiology and ${ }^{2}$ The Harrington Department of Bioengineering, Arizona State University, Tempe, Arizona 85287, and ${ }^{3}$ Dipartimento di \\ Psicologia Generale, Università di Padova, 35131 Padova, Italy
}

It has been shown that when subjects can predict object properties [e.g., weight or center of mass (CM)], fingertip forces are appropriately scaled before the object is lifted, i.e., before somatosensory feedback can be processed. However, it is not known whether subjects, in addition to these anticipatory force mechanisms, exploit the ability to choose where digits can be placed to facilitate object manipulation. We addressed this question by asking subjects to reach and grasp an object whose CM was changed to the left, center, or right of the object in either a predictable or unpredictable manner. The only task requirement was to minimize object roll during lift. We hypothesized that subjects would modulate contact points but only when object CM location could be predicted. As expected, object roll was significantly smaller in the predictable condition. This experimental condition was also associated with statistically distinct spatial distributions of contact points as a function of object CM location but primarily when large torques had to be counteracted, i.e., for right and left CM locations. In contrast, when subjects could not anticipate CM location, a "default" distribution of contact points was used, this being statistically indistinguishable from that adopted for the center CM location in the predictable condition. We conclude that choice of contact points is integrated with anticipatory force control mechanisms to facilitate object manipulation. These results demonstrate that planning of digit placement is an important component of grasp control.

Key words: hand; reaching; kinematics; fingertips; feedback; anticipation

\section{Introduction}

Studies of hand kinematics have shown that the choice of hand placement on an object is highly sensitive to the planned action after object contact, e.g., where the object has to be transported (Cohen and Rosenbaum, 2004). Planned manipulation appears to affect not only where the object is grasped but also how the hand is shaped during the reach (Ansuini et al., 2006). The general interpretation of these results is that the motor plan underlying hand-object interactions takes into account task-specific criteria to enable successful completion of object manipulation, such as maintaining joint angular excursion within a comfortable range (for review, see Rosenbaum et al., 1996). The major limitation of these kinematic studies, however, is that they focus on whole-hand placement relative to the object rather than how placement of individual digits is coordinated. Furthermore, these studies have not examined hand placement in a wide range of behavioral contexts, e.g., the effect of object properties or their predictability.

Given the large number of hand muscles and joints, a given

Received Oct. 27, 2006; revised March 6, 2007; accepted March 7, 2007.

We thank Dr. Thanassis Rikakis, Todd Ingalls, Jodi James, and Siew Wong from Arts, Media, and Engineering for use of their facilities, technical support, data collection, and processing. We thank Drs. Stephen Helms Tillery, Natalia Dounskaia, and Arend Van Gemmert and the Neural Control of Movement Laboratory members for their comments on a previous version of this manuscript. We also thank Emily Kaine, Lance Mollenhauer, Eric Hernandez, and April Reinert for helping with data processing.

Correspondence should be addressed to Marco Santello, Department of Kinesiology, Arizona State University, Tempe, AZ 85287-0404. E-mail: marco.santello@asu.edu.

DOI:10.1523/JNEUROSCI.4693-06.2007

Copyright $\odot 2007$ Society for Neuroscience $\quad$ 0270-6474/07/273894-10\$15.00/0 object manipulation (e.g., translation or rotation) can be performed using many spatial distributions of the digits on the object. At the other extreme, i.e., when contact points cannot be chosen by the subjects, forces at individual digits can be effectively modulated across a wide range of task conditions (Santello and Soechting, 2000; Reilmann et al., 2001; Aoki et al., 2006; Gao et al., 2006). However, the results of studies of force coordination during grasping cannot be used to fully infer the relationship between digit placement on an object and the subsequent force control, because digit placement is constrained by the location of the force sensors. The present study was designed to address the questions of whether and how contact points of individual digits are modulated to object center of mass (CM) location and its predictability. Because changing object CM introduced an external torque, the task requirement was to minimize object roll during lift.

From a biomechanical perspective, our task could have been performed using the same distribution of contact points, regardless of the change in CM location by simply altering the forces at each digit accordingly. However, we hypothesized that certain digit distributions might have allowed for better task performance by facilitating the production of the torque necessary to counteract object roll. Thus, in the predictable CM condition, we expected subjects to modulate the spatial distribution of contact points as a function of object CM location to a greater extent than in the unpredictable condition. We also predicted that such modulation, coupled with the ability to anticipate the forces necessary to counter the external torque, would have led to significantly smaller object rolls. 
A

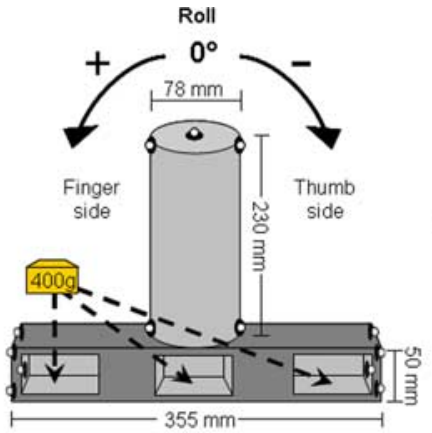

C

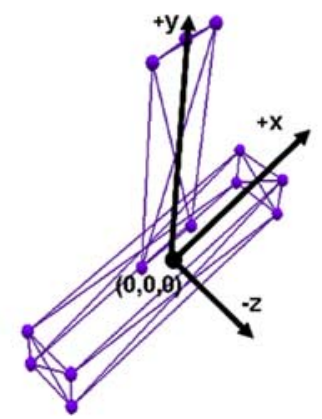

B
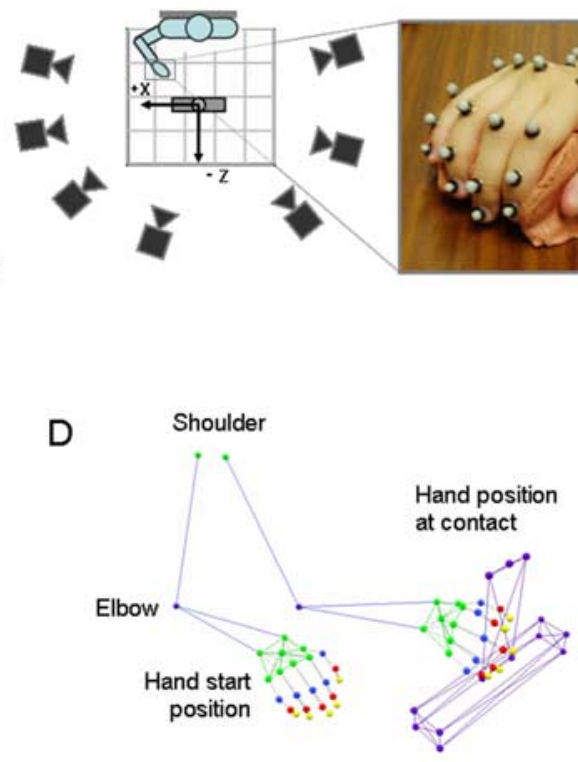

Figure 1. Experimental setup. $\boldsymbol{A}$ shows the object (frontal plane as viewed by the experimenter) used for our reach-to-grasp task, the location of the reflective markers, and the slots where mass was added to change object CM location. Object rolls toward the thumb and finger sides were defined as negative and positive angles, respectively, relative to the vertical $(y)$ in the gravitational frame of reference. $\boldsymbol{B}$ shows the approximate location of the cameras and the workspace used for motion capture (top view; figure is not to scale). The photo shows start hand posture together with marker location on the hand and wrist. ( shows the object model used to measure object translation and rotation. The object was positioned such as to align the origin of the workspace frame of reference with the midpoint of the base of the object. $\boldsymbol{D}$ shows the arm and hand models in the start position and at contact with the object.

\section{Materials and Methods}

Subjects. Twelve subjects (six females and six males, between the ages of 21 and 43 years) took part in the experiments. All participants were right-handed and reported normal or corrected-to-normal vision. All subjects were naive as to the experimental purpose and gave informed consent to participate in the study. The experimental procedures were approved by the Institutional Review Board at Arizona State University and were in accordance with the Declaration of Helsinki.

Experimental task. We asked subjects to reach, grasp, lift, and replace a T-shaped object consisting of a 78-mm-diameter cylinder attached to a horizontal base (Fig. $1 \mathrm{~A}$ ) with their right hand. The height of the cylinder $(230 \mathrm{~mm})$ was chosen to allow a wide range of vertical positions of the fingertips. To verify the extent to which subjects could distribute their fingertips on the object, we measured maximum finger span as the distance between the index and little fingertips with all fingers maximally abducted on a flat surface. Maximum finger span ranged from 82.9 to $149.0 \mathrm{~mm}(109.4 \pm 20.3 \mathrm{~mm}$, average $\pm \mathrm{SD})$, corresponding to $\sim 36$ and $68 \%$ of the height of the cylinder, respectively.

The CM of the object was changed either in a trial-to-trial manner (unpredictable condition) or across blocks of trials (predictable condition; see below) by adding a mass $(400 \mathrm{~g})$ in one of three slots at the base of the object situated $-140,0$, and $+140 \mathrm{~mm}$ from the midpoint of the object base, i.e., left (thumb side), center, and right (finger side) CM locations, respectively (Fig. 1A). The external torques exerted by the added mass relative to the midpoint of the base of the object were -0.549 $\mathrm{Nm}$ (torque toward the thumb side), $0 \mathrm{Nm}$, and $+0.549 \mathrm{Nm}$ (torque toward the finger side) when the weight was added at the left, center, or right slot, respectively (Fig. $1 A$ ). To prevent the added mass from shifting during object lift, we used Velcro to stabilize the added mass in the slot. The total weight of the object with the added mass was $0.81 \mathrm{~kg}$.

For the predictable condition, subjects were informed that object CM location would be the same for the entire block of trials. For the unpredictable condition, subjects were informed that CM location would be changed from trial to trial in random order. For both experimental conditions, subjects were not allowed to view the weight exchanges or the CM location. Note that subjects could not anticipate CM location for the first trial of each block of the predictable condition. Therefore, this trial was considered a practice trial and was not included in the statistical analysis.

The subject sat on a height-adjustable chair in front of a table with the elbow and wrist resting on the table, the forearm pronated, the arm oriented in the parasagittal plane passing through the shoulder, and the right hand in a designated start position (Fig. $1 \mathrm{~B}$ ). In the start position, the hand was pronated with the palm resting on a clay mold that was shaped to allow a comfortable and repeatable posture of all digits, i.e., slightly flexed at the metacarpal and proximal interphalangeal joints (see photo in Fig. $1 B$ ). The object was placed on a table and aligned with the subjects' midline, the object and subject frontal planes being aligned with each other. The object was located at a distance of $\sim 30 \mathrm{~cm}$ and an angle of $\sim 35^{\circ}$ between the sagittal plane of the object and the hand start position. Subjects started the reach-to-grasp movement after a verbal signal from the experimenter. We instructed the subjects (1) to perform the movement at a natural speed, (2) to grasp the object (cylinder) with all digits, and (3) to lift the object vertically to a comfortable height of $\sim 15-20 \mathrm{~cm}$ above the table while trying to maintain its vertical alignment, i.e., to minimize object roll. Naturally, we expected that complying with the third requirement would have been more difficult in the unpredictable condition because of the subjects' inability to anticipate the direction of the external torque. No other instructions were given with regard to how or where the object was to be contacted. During data collection, one of the experimenters visually verified that subjects complied with all task requirements for each trial.

Subjects performed a total of 15 experimental trials, i.e., $3 \mathrm{CM}$ locations $\times 5$ trials, for each experimental condition. For the predictable condition, one block of six trials was administered for each CM location (left, center, and right) consisting of one practice trial (see above), followed by five experimental trials. For the unpredictable condition, CM locations were changed from trial to trial as described above. On average, the time between trials was $15 \mathrm{~s}$. Experimental conditions and CM locations were counterbalanced across subjects.

Recording of hand and object kinematics. To track the kinematics of the hand, we used a near-infrared camera motion capture system (frame rate, $100 \mathrm{~Hz}$; shutter speed, 800 frames/s; Eagle-4 Digital System; Motion Analysis Corporation, Santa Rosa, CA). Light-weight retro-reflective markers (4 and $6 \mathrm{~mm}$ in diameter; Motion Analysis Corporation) were placed on the hand (Fig. $1 B$ ) to create a hand model (Fig. 1D) for kinematic analysis. For all digits, markers were glued to the central region of the nails for the computation and analysis of digit placement on the object. Note that we will refer to these five markers as "fingertips," thus including the tip of the thumb. We also placed markers on all joints of each digit, the dorsal aspect of the hand, the radial and ulnar styloids, the lateral epicondyle of the humerus, and the acromion to record hand and arm kinematics (Fig. 1D). Because the focus of this paper is on the control of contact points, here we report only the analysis of spatial and temporal variables related to the tip of each digit at contact with the object (hand and arm kinematics during the reach will be reported in another paper). Markers were also placed on the object (Fig. $1 \mathrm{~A}$ ) to track its movement after contact and to compute the location of each fingertip relative to the object.

To record the movement, we used seven cameras placed in a semicircle 
at a distance of $1-1.5 \mathrm{~m}$ from the table on which the subject's hand and object were placed (Fig. $1 B)$. To optimize accurate tracking of all markers, the system was calibrated before data collection by first having the subject perform reaching and grasping movements as the experimenter adjusted the camera position, roll angle, zoom, focus, threshold, and brightness. These procedures were followed by static and dynamic calibration. For the static calibration, an L-frame bracket of four markers at known distances from each other was placed in the middle of the table. For the dynamic calibration, a three marker wand was moved throughout the workspace of interest for $\sim 60 \mathrm{~s}$. The origin of the frame of reference of the workspace coincided with that of the frame of reference of the object (Fig. 1C). The spatial accuracy of the recording system was $\sim 0.15 \mathrm{~mm}$ in the $x, y$, and $z$ planes.

Data processing. After data collection, each trial was individually checked for correct marker identification and then run through a low-pass Butterworth filter with a $6 \mathrm{~Hz}$ cutoff. For data processing and analysis, we wrote custom software (Matlab; MathWorks, Natick, MA) to compute the following temporal and spatial variables.

(1) "Reach onset" was defined as the time at which the tangential velocity of the radius marker crossed a threshold $(5 \mathrm{~mm} / \mathrm{s})$ and remained above it for longer than $500 \mathrm{~ms}$ (Fig. $2 \mathrm{~B}$ ).

(2) "Object lift onset" was defined as the time at which the tangential velocity of the top center marker of the object (Fig. $1 \mathrm{~A}$ ) crossed a threshold $(5 \mathrm{~mm} / \mathrm{s})$ and remained above it for longer than $200 \mathrm{~ms}$ (Fig. 2). Note that our definition of object lift onset temporally coincides with the end of the reach as defined in previous studies (Santello and Soechting, 1998; Ansuini et al., 2006).

(3) "Reach duration" was computed as the time interval between reach onset and object lift onset (Fig. 2B).

(4) "Digit contact time" was quantified as the time at which the digit made contact with the object relative to reach onset and defined as the time at which the tangential velocity of the marker of the tip of the digit reached its minimum value (Fig. $2 C$ ). The search for the minimum tangential velocity of each fingertip was performed from the time of peak wrist velocity (marker on the radius) to object lift onset. Note that, for virtually all trials ( $>98 \%$ ), subjects made contact with the object with all digits before object lift as visually assessed by one of the experimenters and as verified off-line (see below). Also note that the spatial location of each fingertip (see below) was measured at the estimated contact time. For each trial, we verified accuracy of the above algorithm to determine digit contact time off-line by using measures computed from the object model (cylinder diameter) and the hand model (distance between the thumb and the digits). We found that fingertip tangential velocity accurately defined the time of contact between the fingertip and the object.

(5) "Grip aperture" was computed as the distance between the threedimensional coordinates of the thumb and each of the fingers at time of contact.

(6) "Fingertip contact points" were defined as the $y$ - and $z$-coordinates (vertical and anteroposterior dimensions, respectively) of the fingertip on the object at contact time. Because the graspable part of the object was limited to the cylinder, the $y$-coordinate was defined as the vertical distance (in millimeters) between the fingertip and the base of the cylinder. The contact point $z$-coordinate refers to the perpendicular distance between the fingertip and the horizontal axis of the object (Fig. 1C, $x$-axis). Note that the third dimension $(x)$ of the fingertip coordinate at contact with the object is redundant because it covaries with the $z$-coordinate.
(7) "Contact duration" was defined as the time interval between the first and last digit contacting the object.

(8) "Pre-lift duration" was defined as the time interval between the last digit contacting the object and object lift onset.

(9) "Object roll" was defined as the angle between vertical and the line connecting top and bottom markers of the object. The focus of our behavioral analysis was on the initial roll occurring shortly after object lift onset. This epoch was used to measure the greatest roll occurring as a result of erroneously planned fingertip forces, i.e., before corrective responses countering object roll can be made at reaction time latencies. We first measured peak object roll throughout the entire object lift. We then verified the accuracy of this algorithm by inspecting individual trials. We found that the peak rolls occurred very early after object lift onset $(\sim 240$ $\mathrm{ms}$ ) (see Fig. $8 \mathrm{~A}$ ). These latencies are comparable with those reported by Salimi et al. (2000) of $\sim 176 \mathrm{~ms}$ for two-digit grasp. However, in a few cases, particularly for the center CM location and in the predictable condition, the greatest roll was small (less than $\pm 3^{\circ}$ ) and occurred as a result of overcompensation of the corrective response, hence later during the lift. For these cases, peak object roll was defined as the initial roll (occurring within $500 \mathrm{~ms}$ from object lift onset) with a magnitude $>1^{\circ}$.

Statistical analysis. For the object to be lifted vertically, the external torque generated by the added mass had to be counteracted by a net torque of equal magnitude and opposite direction resulting from forces exerted by all digits. It should be emphasized that the ability to anticipate object CM location (predictable condition) allows subjects to predict the digit forces required to minimize object roll before lifting the object. In contrast, for the unpredictable condition, object CM location can only be perceived after the object has been lifted (Rearick and Santello, 2002; Santello et al., 2004). In protocols such as ours, evidence for anticipatory force control mechanisms is provided by smaller object roll when performing the task in the predictable CM condition (Salimi et al., 2000). The objective of this study was to assess whether subjects, in addition to using the anticipatory force control mechanisms cited above, would 
implement a strategy based on modulation of digit contact points. Specifically, subjects could have tried to minimize object roll using two alternative strategies: (1) use the same contact points regardless of the external torque, or (2) use distinct spatial distributions of digit contact points as a function of object CM location. The implication associated with using strategy 1 is that subjects would resort to modulate contact forces at individual digits to keep a vertical object orientation (as described by studies in which no modulation of contact points on the object is allowed) (see Introduction). Evidence for strategy 2, however, would imply that force modulation is planned in conjunction with the selection of object CM-specific distributions of contact points. Note that subjects could have used strategy 2 only when object CM location could be predicted on a trial-to-trial basis.

To distinguish between the above two strategies, we assessed the effect of object CM location and its predictability on reach and grasp kinematics in the temporal and spatial domain. For the temporal analysis we analyzed the following: (1) reach duration, (2) digit contact time, (3) contact duration, and (4) pre-lift duration. For the spatial analysis, we analyzed (5) grip aperture and (6) fingertip contact point(s) in three different ways: (a) for each digit (ANOVA, with repeated measures; see below), (b) for digit pairs and, (c) for the five-digit spatial distribution.

For digit pair analysis, we performed linear regression analysis to quantify the extent to which the $y$-coordinate of the contact point of a given digit covaried with the $y$-coordinate of another digit. Because the degree of movement independence of the digits is limited (for review, see Schieber and Santello, 2004), the extent to which a digit contact point can be selected independently from another is also limited. At one extreme, subjects might have chosen to modulate the vertical location of the whole hand (Rosenbaum et al., 1996) while maintaining the distance between fingertips. This would have resulted in a perfect linear covariation between all digit contact points. At another extreme, the strength of covariation between all digit pairs might have been modulated to CM location and/or predictability condition. An intermediate scenario would consist of a task-dependent modulation of covariation patterns in some but not all digits. Linear regression analysis was performed on data from all CMs pooled into a single data group. Correlation coefficients were $z$-normalized before averaging.

For the analysis of the spatial distribution of all digits, we performed discriminant analysis to quantify the extent to which spatial distributions of all contact points $(n=5)$ can be distinguished both within a set of trials performed for a given object CM location and between groups of trials associated with each CM location. This analysis was performed separately for each predictability condition (for more details, see Santello and Soechting, 1998; Santello et al., 2004). Briefly, we computed discriminant functions that maximized the ratio of the between-group variance to the within-group variance (Johnson and Wichern, 1992), larger ratios denoting stronger relationships between object CM location and spatial distribution of contact points. The output of this analysis determines the extent to which a priori membership of each trial to a given CM location (i.e., five trials per $\mathrm{CM}$ location) is accurately predicted from the data by generating a confusion matrix (Sakitt, 1980; Johnson and Phillips, 1981). To quantify the relationship between predicted versus actual trial membership, we used information theory (Shannon, 1948) to compute the sensorimotor efficiency (SME) index (Sakitt, 1980). Perfect discrimination of all contact points as a function of object CM location would result in three distinct data groups, this discrimination corresponding to SME of $100 \%$. In contrast, lack of such relation would correspond to SME of $0 \%$. A less than perfect discrimination would result in intermediate values of SME, indicating that fewer than three CM locations could be discriminated by contact point distribution.

Last, we quantified performance by measuring peak object roll. For qualitative analysis and for graphical purposes (see Fig. 8), information about the directionality of the roll was preserved by expressing the angles relative to the vertical (see above). Note that symmetrical rolls expressed as relative angles (for instance, $+3^{\circ}$ and $-3^{\circ}$; see above convention) are identical in terms of roll magnitude, hence performance error, but statistically might appear as significantly different (i.e., a $6^{\circ}$ difference). To prevent this confound, statistical analysis was performed on absolute roll angles.
For each of the above temporal and spatial variables, we performed ANOVA with repeated measures with "experimental condition" (predictable vs unpredictable) and "CM location" (left, center, and right) as the within-subject factors. Main effects were used to explore the means of interest (post hoc $t$ test), and Bonferroni's corrections ( $\alpha$ level of $p<$ $0.05)$ were applied.

\section{Results}

\section{Reach duration}

Reach duration was significantly longer in the predictable than in unpredictable condition (1204 vs 1152 ms, respectively; main effect of experimental condition, $F_{(1,59)}=6.491$, $\left.p<0.05\right)$. Object $\mathrm{CM}$ location also affected the duration of the reach, i.e., the movement duration was shorter for reaches to the object with $\mathrm{CM}$ in the right than in the center location (1145 vs $1206 \mathrm{~ms}$, respectively; main effect of CM location, $F_{(2,118)}=4.889, p<$ $0.001)$. Although reach duration was affected by CM location more in the predictable than unpredictable condition (experimental condition $\times \mathrm{CM}$ location, $\left.F_{(2,118)}=4.178, p<0.05\right)$, none of the post hoc comparisons reached the level of significance ( $p$ values $>0.05)$.

\section{Contact time of the digits}

Overall, the effects of object CM location and its predictability on the temporal control of contact points were limited to only the little finger. This finger contacted the object later in the predictable than in the unpredictable condition (1088 vs $1048 \mathrm{~ms}$, respectively; multivariate ANOVA; main effect of experimental condition, $\left.F_{(1,59)}=5.212, p<0.05\right)$. However, the betweencondition difference in mean contact time was very small. The index finger tended to contact the object later when the CM was in the center than when it was in the right location (1081 vs 1041 ms, respectively; main effect of CM location), but this effect did not reach significance $(p=0.057)$.

\section{Contact and pre-lift duration}

The ANOVA performed on contact duration (i.e., the time between the first and last digit contact time; see Materials and Methods) did not reveal a significant effect of either experimental condition or CM location. Although the two-way interaction between these two factors was significant $\left(F_{(2,118)}=3.940, p<\right.$ $0.05)$, post hoc analyses revealed no significant differences. The same analysis performed on the time between the last digit contacting the object and object lift onset (i.e., pre-lift duration; see Materials and Methods) revealed only a significant two-way interaction, experimental condition $\times \mathrm{CM}$ location $\left(F_{(2,118)}=\right.$ 4.841, $p<0.01$ ). Post hoc comparisons showed that this time interval was not significantly different among CM locations in the predictable condition (left, center, and right CM: 86, 73, and 68 $\mathrm{ms}$, respectively). For the unpredictable condition, however, prelift duration was significantly longer for the center CM location $(80 \mathrm{~ms})$ than for the right and left CM locations (43 and $58 \mathrm{~ms}$, respectively). Last, pre-lift duration for left and right $\mathrm{CM}$ locations in unpredictable trials was shorter than that for the left CM location in predictable trials.

\section{Grip orientation and aperture}

The distribution of contact points is illustrated in Figure 3. For clarity, contact points in three dimensions are shown in two views $(x-z$ plane and $y-z$ plane) to depict both the grip aperture (see Materials and Methods) and orientation relative to the forearm and object at contact. Data are presented from one representative subject but portray the general trends shared by the entire pool of 
subjects. In the predictable condition, this subject modulated contact point distributions as a function of CM location but only for the predictable condition. However, this modulation occurred primarily along the vertical axis of the object (i.e., $y-z$ plane) rather than around the circumference of the object (i.e., $x-z$ plane). Specifically, the thumb was placed higher on the cylinder when the CM was located on the left (thumb side) of the object and lower when the CM was on the right (finger side). A reciprocal pattern is apparent for the index finger. In contrast, grip orientation was not greatly affected, with alterations in orientation mostly occurring as a result of changes in thumb contact point (compare left with center and right CM for the predictable condition in Fig. 3).

In addition to modulating contact points, Figure 3 shows that this subject also changed wrist pronation/supination angle across CM locations in the predictable condition. This suggests that wrist rotation may also be controlled in an anticipatory manner to minimize object roll. This parallels the reciprocal modulation of the vertical position of the contact points of the thumb and index in the predictable condition as a function of CM location. Specifically, when the CM was on the left, the thumb was higher and the wrist more supinated, and vice versa when CM was on the right. This preliminary result was observed across all subjects and will be analyzed in the context of the coordination between wholearm kinematics and contact points in a subsequent paper.

To determine the effect of CM location and its predictability on the distance between the thumb and each finger at contact, we performed an ANOVA on grip aperture (see Materials and Methods) (Fig. 3). A main effect was found for both experimental condition and CM location $(p<0.01)$. Also, the two-way interaction between experiment condition and CM location was significant for thumb-index, thumb-ring, and thumb-little finger pairs $\left(F_{(2,118)}=34.241, p<0.0001 ; F_{(2,118)}=26.463, p<0.0001\right.$; and $\mathrm{F}_{(2,118)}=54.218, p<0.0001$, respectively). No interaction of thumb-middle finger pair was found.

The post hoc analysis of the digit pairs revealed significant differences for all of the thumb-finger pairs (except thumb-middle) in the predictable condition between the left CM location and both the center and right CM locations. The thumb-little finger pair also exhibited differences between the center and right CM locations. However, there were no significant differences between CM locations in the unpredictable condition for all digit pairs. The differences in grip aperture found in the predictable condition stem from the changes in the spatial distribution of the fingertips, which are further examined below by analyzing the modulation of contact points across predictability conditions.

\section{Contact points of individual digits}

Because changes in $\mathrm{CM}$ location were designed to generate torques within the vertical plane only (Fig. $1 C, x-y$ plane), minimization of object roll required subjects to generate counteracting torques within the same plane. If subjects had chosen to modulate contact points to assist digit force modulation, we would have expected this to occur primarily on the vertical dimension

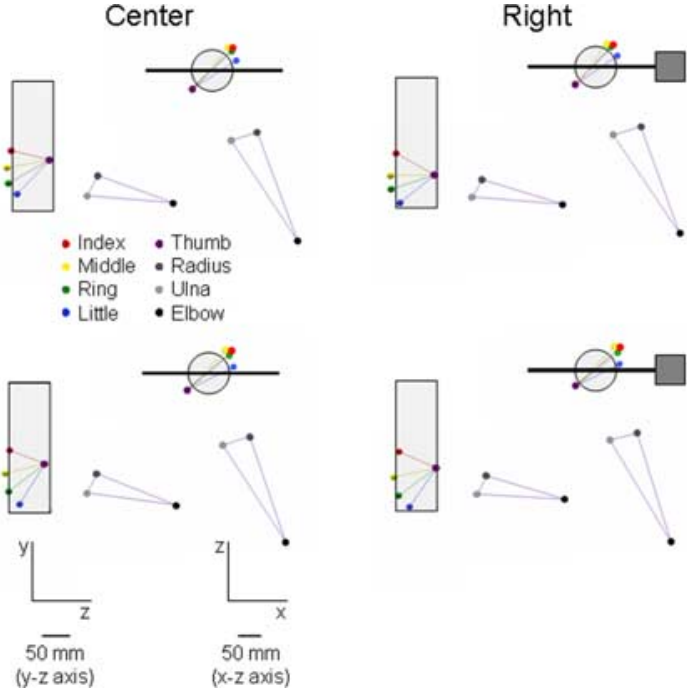

and significantly less in the anteroposterior dimension. This expectation was met because the maximum modulation among all fingertips in the anteroposterior dimension was small, i.e., from $2.9 \mathrm{~mm}$ (thumb) to $5.7 \mathrm{~mm}$ (little finger). In contrast, maximum modulation of contact points in the vertical dimension was from $5.1 \mathrm{~mm}$ (ring finger) to $23.4 \mathrm{~mm}$ (thumb), i.e., on average a fivefold difference in the vertical versus anteroposterior maximum modulation of contact points. Therefore, all analyses presented below were performed on the vertical fingertip position.

Vertical location of the tip of each digit on the object (i.e., fingertip contact points; see Materials and Methods) was modulated as a function of object CM location but only when CM could be predicted on a trial-to-trial basis. Specifically, in the unpredictable condition, subjects chose to use a default distribution of contact points on the object, the maximum modulation of fingertip locations ranging from $1.1 \mathrm{~mm}$ (thumb) to $4.0 \mathrm{~mm}$ (index). In the predictable condition, however, we found larger changes in contact points across CM locations, the maximum modulation ranging from $3.8 \mathrm{~mm}$ (ring finger) to $23.4 \mathrm{~mm}$ (thumb). For all digits, we found a significant interaction between experimental condition and CM location (i.e., thumb, $F_{(2,118)}=21.630, p<0.0001$; index finger, $F_{(2,118)}=19.526, p<$ 0.0001 ; middle finger, $F_{(2,118)}=6.983, p<0.01$; ring finger, $F_{(2,118)}=4.775, p<0.05$; little finger, $\left.F_{(2,118)}=6.471, p<0.01\right)$.

For the thumb, post hoc comparisons revealed that, in the predictable condition, this contact point was significantly higher when the object CM was located in the left $(121.4 \mathrm{~mm})$ than when it was in the center or in the right (104.8 and $98.0 \mathrm{~mm}$, respectively) (Fig. 4). When comparing predictable and unpredictable conditions, a significant difference in thumb contact point was found only for the left CM location. An opposite pattern was found for the index finger, post hoc comparisons revealed that, in the predictable condition, this contact point was significantly lower for the left than for the right CM location (132.4 and 145.7 $\mathrm{mm}$, respectively) (Fig. 4). In the unpredictable condition, CM 


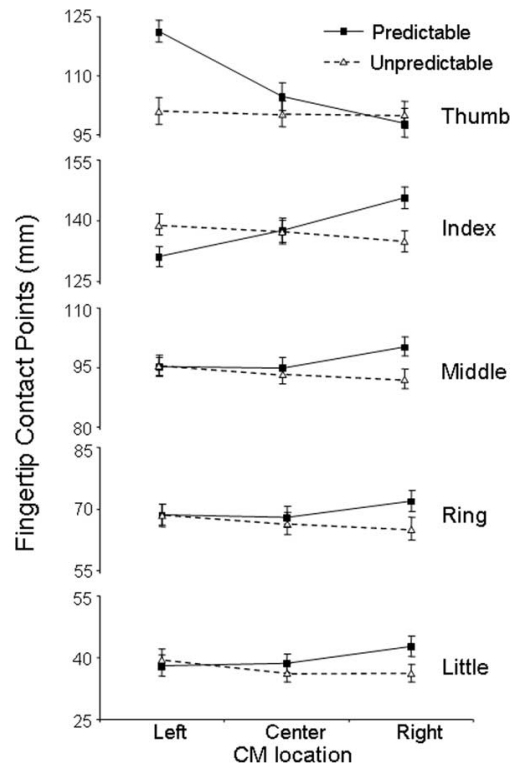

Figure 4. Individual fingertip contact points. Fingertip vertical location relative to the base of the cylinder is shown for each digit as a function of CM location and predictability condition. The scale of the vertical axes is the same for all plots to allow comparison across digits. Data are means \pm SE of all subjects.

location did not significantly affect either thumb or index finger contact points.

For the middle, ring, and little finger contact points, post hoc comparisons of the interaction experimental condition $\times \mathrm{CM}$ location revealed no significant differences. Nevertheless, a trend was found for the contact points of these digits. Inspection of the contact point distributions for the predictable condition revealed that subjects tended to place these digits higher for the right than for left and center CM locations. This trend, however, was not found when subjects reached to grasp in the unpredictable condition (Fig. 4).

\section{Covariation between pairs of fingertip contact points}

We performed linear regression analysis to quantify the extent to which the contact point of a given digit was associated with that of another digit and how these relationships were affected by predictability of CM location (note that this analysis was performed on trials pooled from all CM locations; see Materials and Methods). As one would expect, the extent to which given pairs of contact points covaried differed across subjects, these differences probably resulting from a combination of factors such as hand size and idiosyncratic patterns of hand postures on the object. For example, slopes of the relationships between thumb and index finger contact points (i.e., the digits that were characterized by the largest modulation) (Fig. 4) for the predictable condition ranged across all subjects from -0.33 to $+0.75 \mathrm{~mm}$ (subjects 2 and 9, respectively).

Figure $5 \mathrm{~A}$ shows the relationship between contact points for the thumb versus index finger, thumb versus little finger, and middle versus ring finger from a representative subject (subject 10 ) in both the predictable and unpredictable conditions. For this subject, unpredictable CM trials were characterized by stronger linear relationships than those from the predictable CM condition, although this difference was found only for specific digit pairs, e.g., thumb versus index and thumb versus little finger relationships. For these digit pairs and for the predictable condition, variations in thumb contact points were not systematically related to variations in either index or little finger contact points, the maximum amount of variance accounted for being $<30 \%$. Note that for these two digit pairs, the thumb contact point is modulated to object CM location to a greater extent than either the index or little finger.

Figure $5 B$ shows the magnitude of correlation coefficients averaged across all subjects for each digit pair and predictability condition. For the predictable condition, all thumb-finger pairs were characterized by the highest degree of independence, i.e., the lowest $r$ values. Across finger pairs, adjacent fingers tended to exhibit less independence than nonadjacent fingers, the middle and ring fingers being the least independent finger pair. The extent of adduction/abduction between digits was modulated to object CM location in a digit pair-specific manner. Furthermore, between-digit relationships for some digit pairs were sensitive to whether object CM location could be predicted or not. In the unpredictable condition, subjects adopted hand postures characterized by a lower degree of independence between individual contact points as revealed by the tendency for stronger linear relationships than in the predictable condition.

\section{Coordination of multidigit contact points}

Individual digit data presented in Figure 4 are plotted together in Figure 6 to illustrate how the whole hand was placed on the object. On average, the highest and lowest contact points for the predictable condition were 145.7 and $38.1 \mathrm{~mm}$ (index and little finger, respectively), equivalent to a vertical finger span of 107.6 $\mathrm{mm}$. The unpredictable condition was associated with a slightly smaller finger span $(102.9 \mathrm{~mm})$ and lower index and little finger contact points (139.1 and $36.3 \mathrm{~mm}$, respectively). As pointed out above, the effect of predictability of CM location is significantly clearer for right and left than for center CM location, the latter being characterized by greater overlap of data from the two experimental conditions (predictable vs unpredictable).

To quantify the extent to which a given five-contact point distribution could be predicted by object CM location, we performed discriminant analysis (see Materials and Methods). Figure $7 A$ shows the allocation of each trial to each $C M$ location in discriminant space defined by the coefficients of the first two discriminant functions (subjects 6 and 10, top and bottom rows, respectively). For the predictable condition (Fig. $7 A$, left column), the distribution of all contact points tended to cluster as a function of object CM location. Visual inspection of the data clusters further indicates that the center CM location tended to be at an intermediate statistical distance between left and right CM locations. Therefore, these two subjects tended to use a consistent distribution of contact points across trials within each CM location yet different across predictability conditions. Note that the distance in discriminant space between trials from left versus right $\mathrm{CM}$ locations is greater than that between either of these and the trials from the center CM location.

When subjects could not anticipate object CM location, contact point distributions could not be discriminated to the same extent as in the predictable condition (Fig. $7 A$, right column), as indicated by the larger overlap of trials in discriminant space. This indicates that these subjects tended to use similar contact point distributions across all CM locations. Low discriminability of contact point distribution across CM locations was found in all but one subject (subject 3 ), this subject exhibiting a weak modulation of contact points in the predictable condition.

To quantify the extent to which spatial distributions of contact points could be discriminated as a function of CM location, we computed the SME index (see Materials and Methods). The pre- 
dictable condition was characterized by a significantly higher SME index than the unpredictable condition $(54.5 \pm 7.1$ vs $13.2 \pm 2.8$, mean $\pm \mathrm{SE}$, respectively; $p<$ 0.001 , two-tailed $t$ test). These values correspond to a discrimination of $\sim 1.8$ and 1.2 CM locations for the predictable and unpredictable condition, respectively. Note that, in the predictable condition, the discrimination of contact points as a function of CM locations was less than perfect, i.e., subjects adopted less than three distinct spatial distributions of contact points. This is consistent with the observation of a clearer discrimination of contact point distributions for right versus left $\mathrm{CM}$ locations, the center CM location being associated with less consistent spatial distributions of contact points (Fig. 6). For the unpredictable condition, virtually no discrimination of contact point distributions was found, the average number of object CM locations that could be predicted by contact point distributions being close one.

The main results of our temporal and spatial analysis of contact points suggest that object CM location and/or its predictability had little or no effect on temporal variables but had significant effects on spatial variables. This indicates that the spatial distribution of contact point is controlled independently from the temporal evolution of digit motion during the reach.

\section{Object roll}

Subjects were able to minimize object roll during lift significantly better when they could anticipate object CM location. Figure $8 \mathrm{~A}$ shows object roll occurring when lifting the object during each experimental condition and CM location for one representative subject (subject 9). As expected, when object CM location could not be anticipated on a trial-to-trial basis, subjects were unable to initially counteract the external torque generated by the added mass, i.e., the object rolled toward its CM location to a greater extent than for the predictable condition (Fig. $8 \mathrm{~A}$, bottom vs top row). The effect of CM location predictability was particularly clear for left and right $\mathrm{CM}$ locations, i.e., when object $\mathrm{CM}$ was distributed asymmetrically relative to the grasped cylinder. When object CM location could be anticipated, however, this subject could minimize object roll consistently across trials and regardless of CM location.

The pattern shown in Figure $8 \mathrm{~A}$ was representative of all subjects. ANOVA performed on peak object roll revealed a significant two-way interaction, experimental condition $\times \mathrm{CM}$ location $\left(F_{(2,118)}=17.526, p<0.0001\right)$. Post hoc exploration of this interaction revealed that, in the predictable condition, no signifhere for the sake of clarity.

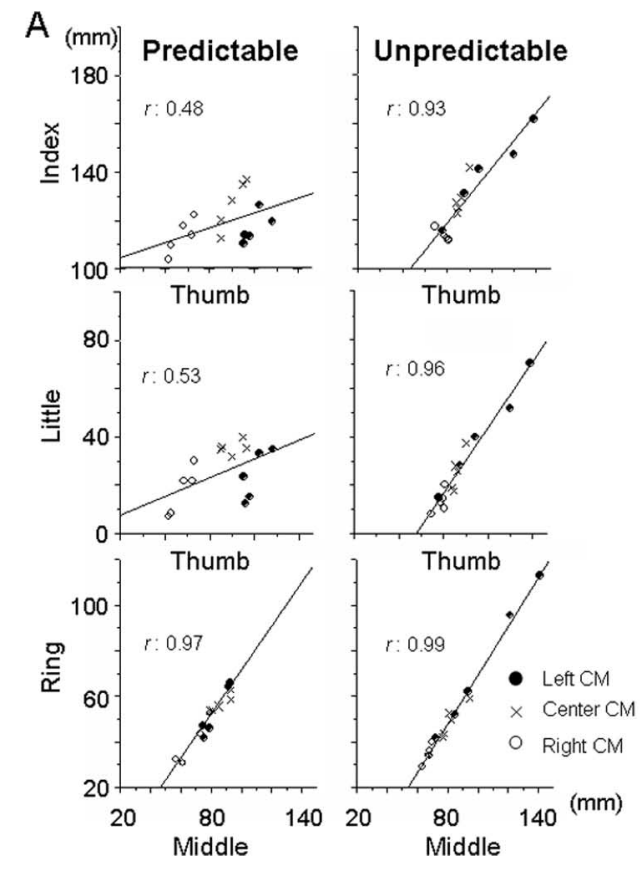

B Predictable

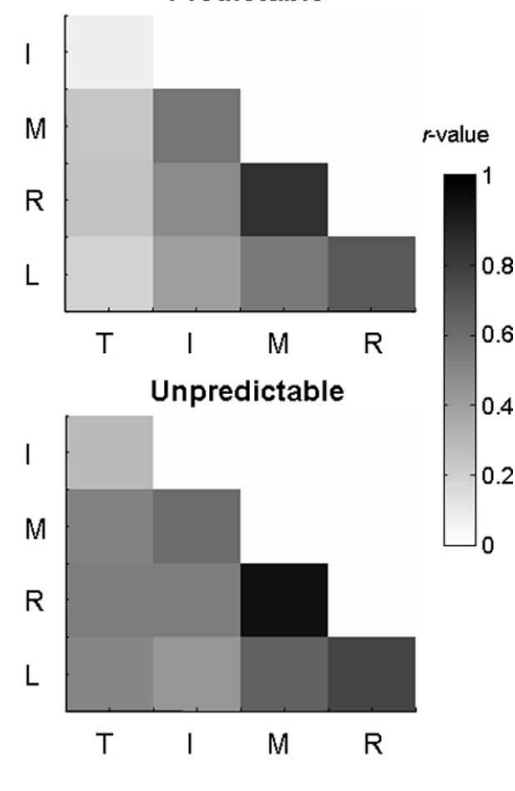

Figure 5. Linear covariation between fingertip contact points. $\boldsymbol{A}$ shows the relationship between contact points of three digit pairs from one subject (subject 10) for each CM location and predictability condition. The magnitude of the correlation coefficient (Pearson's $r$ ) is shown in each plot. $\boldsymbol{B}$ shows the $r$ values of the linear relationships between contact points for each digit pair averaged across all subjects. $T, I, M, R$, and $L$ denote thumb, index, middle, ring, and little fingers, respectively.

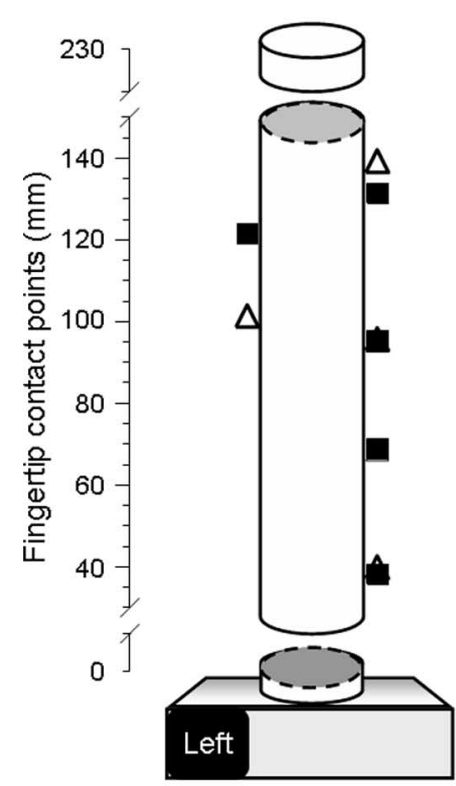

Predictable

$\Delta$ Unpredictable

Figure 6. Spatial distributions of all digits as a function of object CM location and its predictability. The vertical locations of fingertip contact points, shown separately for each digit in Figure 4, are plotted together to show the relationship between whole-hand placement on the object as a function of CM location and predictability condition. SEs (shown in Fig. 4) are not shown

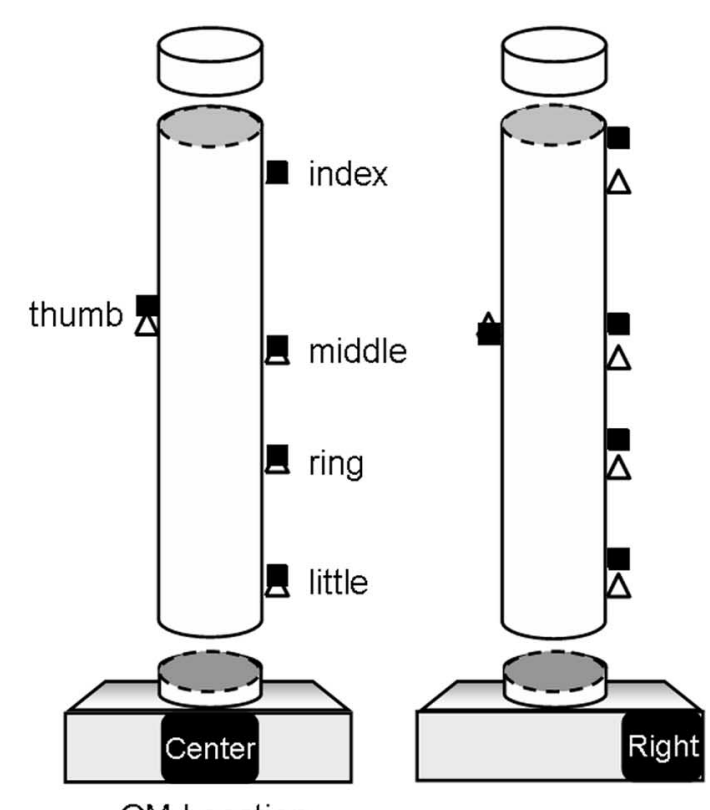

icant differences were found in the absolute magnitude of peak object roll as a function of CM location (left, $2.7^{\circ}$; center, $1.7^{\circ}$; right, $\left.3.4^{\circ}\right)$. In contrast, in the unpredictable condition, peak object roll was greater when the CM location was in either the left or the right $\left(7.8^{\circ}\right.$ and $8.3^{\circ}$, respectively) than when it was in the center $\left(3.2^{\circ}\right)$. Furthermore, the magnitude of peak object roll in 


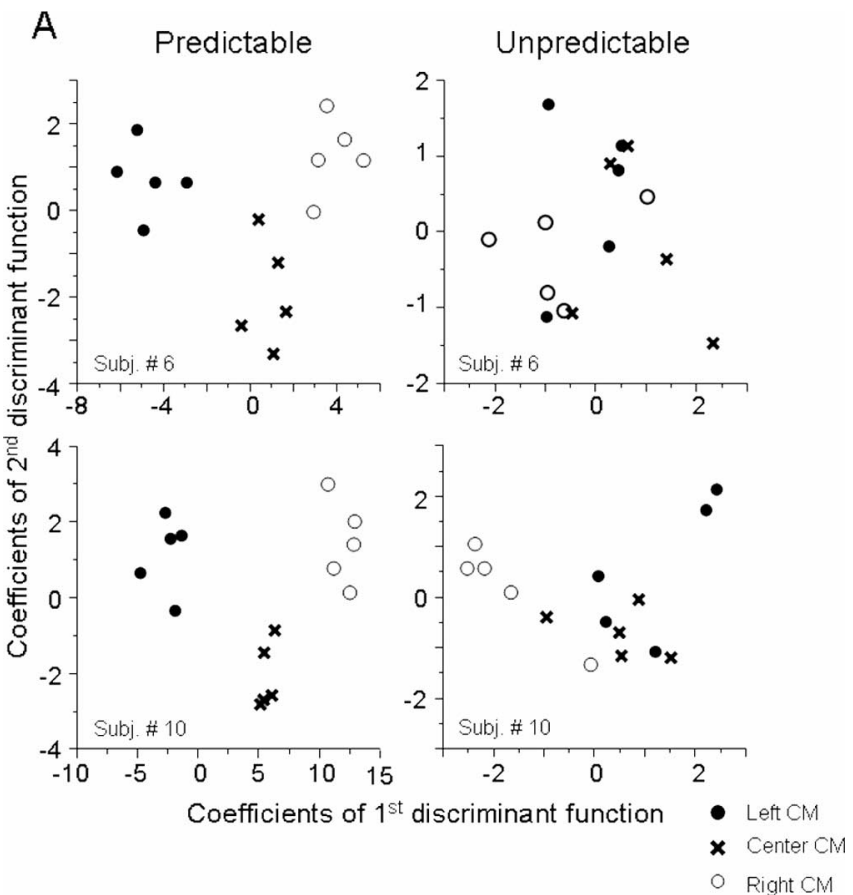

Figure 7. Discrimination of multidigit contact points as a function of object CM location and its predictability. The discrimination of all contact points as a function of object CM location for each predictability condition (left and right column plots) for two representative subjects (subjects 6 and 10, top and bottom rows, respectively). Each trial is plotted in discriminant space defined by the coefficients of the first two discriminant functions.

unpredictable trials for either left or right CM locations was greater than that elicited by any object CM location in the predictable condition (Fig. 8 B). Therefore, in the unpredictable condition, subjects were able to minimize object roll to the same degree as when they could anticipate object CM location but only when object $\mathrm{CM}$ was located in the center, i.e., when there was no external torque generated by the added mass. This result is consistent with the observation that, in the unpredictable condition, the object was grasped as if the object CM were in the center (Figs. $4,6)$.

\section{Discussion}

Subjects were able to minimize object roll during lift to a significantly greater extent when object CM location could be predicted on a trial-to-trial basis. This implies that subjects were able to anticipate the digit forces necessary to counter the external torque caused by the added mass. The predictable CM condition was also associated with a systematic modulation of fingertip contact point distribution on the object. When object CM location could not be anticipated, however, subjects chose a default spatial distribution of contact points and were unable to prevent significant object roll. These results indicate that, if anticipatory force control mechanisms for grasping can be used, they are implemented via a parallel modulation of hand posture relative to the object. Here we discuss the spatial analysis only, because temporal analysis revealed weak or no effects of CM location or its predictability.

\section{Kinematics and kinetics of grasp control}

For the predictable CM condition, subjects chose to modulate the spatial distribution of digit contact points as a function of object CM location, particularly when mass was added to the left or right of the object (Figs. 4, 6). It should be emphasized that this result could not have been predicted based on literature from digit force coordination during grasping. Specifically, when subjects are asked to align an object vertically against large external torques, they can successfully perform this task by modulating forces at individual digits through the same contact points (Shim et al., 2005; Gao et al., 2006) (for review of previous studies, see Zatsiorsky and Latash, 2004). This suggests that subjects could have successfully performed our task without altering fingertip contact point distribution but nevertheless chose to do so. Before addressing the functional role of such modulation, we will first examine how the spatial distribution of contact points was modulated and the effect of a priori knowledge of object CM location.

\section{Modulation of contact points of individual digits and digit pairs}

When contact point modulation was examined on a digit-todigit basis (Fig. 4), we found that subjects responded to changes in object CM location only in the predictable condition and primarily by varying thumb and index finger contact points, whereas the other digits exhibited smaller variations. This might be attributable to the larger force production capability exhibited by the thumb and index finger relative to other digits (Kinoshita et al., 1995; Santello and Soechting, 2000). Hence, careful placement of these two digits might have been functionally more important for preventing object roll during lift.

For a given height of the hand relative to the object, changes in the vertical location of the contact point required adduction or abduction of a digit relative to another digit. However, the extent to which a digit can generate adduction/abduction movement or forces independently from other digits is limited, the thumb and index finger being characterized by the greatest degree of independence (Lang and Schieber, 2004; Reilly and Hammond, 2006). Therefore, differences in the degrees of independence in contact point placement reported here (Fig. $5 B$ ) might have reflected these differences in the degree of movement and/or force independence among digits. However, covariation between contact points of some digit pairs became stronger when object CM location was unpredictable. For this condition, constraining the location of a digit contact point as a function of another could have been an attempt to reduce the number of independent "variables" to be controlled and to ensure repeatability of digit placement across trials. This strategy would have been particularly important in the unpredictable condition because "errors" in the appropriateness of digit placement, combined with the inability to anticipate the necessary forces, might have resulted in very large object rolls. This interpretation is consistent with little or no modulation of contact points in the unpredictable CM condition by individual or multiple digits (Figs. 4, 7, respectively).

\section{Control of whole-hand contact points}

It should be emphasized that the "net" response to changes in object CM location, i.e., minimizing object roll, results from careful positioning of all digits followed by force production. We found that the spatial distribution of all digits could be reliably predicted when object CM location could be anticipated, particularly in the left and right CM locations (Fig. 7).

The effect of planned manipulation on grip type and hand placement has been described for tasks requiring object rotation (for review, see Rosenbaum et al., 1996) and transport (Cohen and Rosenbaum, 2004). It has been suggested that subjects' choice of hand placement on an object takes into account the posture of the limb at the end of the movement to maintain joint angular excursions within a comfortable range, i.e., "end-state 
comfort effect" (Rosenbaum et al., 1992; Short and Cauraugh, 1997; Cohen and Rosenbaum, 2004). Our results extend these observations in a number of ways. First, we have shown that a priori knowledge of object properties plays an important role in the selection of contact points. It is likely that anticipation of torque direction might also induce changes in limb posture, e.g., the degree of forearm pronation/supination at object contact shown in Figure 3. Second, our task allowed us to examine the interaction between anticipatory force and position control by quantifying the behavioral consequences associated with the ability to anticipate the object CM location. Last, we have described between-digit coordination patterns underlying the modulation of contact points of all digits.

The center CM location did not generate an external torque; therefore, it was not as challenging as the other two CM locations to maintaining vertical orientation of the object (Fig. 8). Consequently, it might not be surprising that the relationship between the spatial distribution of contact points of the center CM location in the predictable condition was not as strong as that for the left and right CM locations (Fig. 7). An important observation, however, was that contact point distributions
A

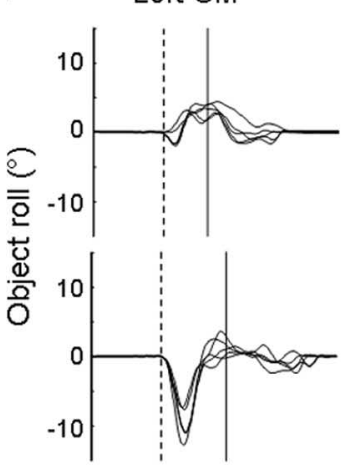

B

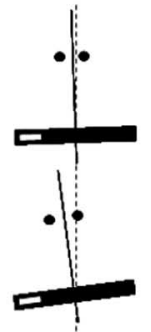

Center CM
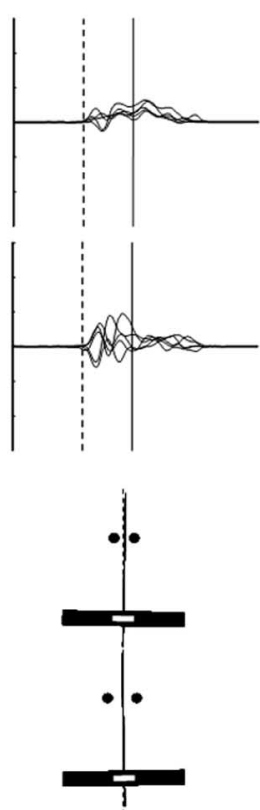

Right CM

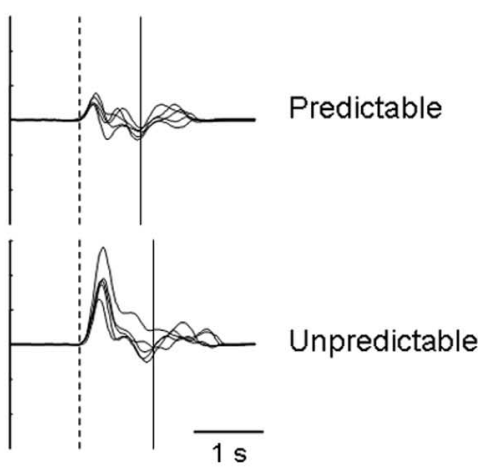

$1 s$
Figure 8. Object roll. A shows the time course of object roll for one representative subject (subject 9). Vertical dashed and solid lines denote object lift onset and end, respectively. All trials are shown for each CM location on the left, center, and right column. Data from predictable and unpredictable conditions are shown on the top and bottom rows, respectively. Data are aligned with respect to object lift onset. $\boldsymbol{B}$ shows average peak object roll (solid line) $\pm \mathrm{SE}$ (filled circles) relative to the vertical (dashed line) as a function of CM location (columns) and predictability condition (rows). For graphical purposes, object roll relative to the vertical was averaged across subjects (solid lines) to preserve information about roll direction. Note that average relative roll shown in the figure does not coincide with the average absolute roll reported in text (for details of this statistical analysis, see Materials and Methods).

for the center CM location in the predictable condition was statistically indistinguishable from those adopted when grasping the object at all CM locations in the unpredictable condition (Figs. 4, 6, 7A). This default strategy appears to be optimal when attempting to minimize roll without knowing object CM location. Adopting a "wrongly" anticipated contact point distribution appropriate for left or right CM locations would have been detrimental if object CM were in the right or left locations, respectively, i.e., subjects would have exerted a torque in the same direction as that generated by the added mass. In contrast, placing the digits as if preparing to lift the object with a center CM location would have minimized the effects of such errors. Finally, if the actual CM location was in fact located in the center, subjects would have had little trouble in minimizing object roll (Fig. 8, Unpredictable rows). An additional explanation for a bias toward a "center CM distribution" of contact points might be that, when humans interact with symmetrical objects such as the cylinder used in our task (i.e., a bottle or a can), their center of mass is also expected to be symmetrical relative to their graspable surfaces.

\section{Anticipatory control mechanisms for grasping}

Using a similar object CM manipulandum as the one used here, Salimi et al. (2000) reported that, after a few lifts, subjects were able to asymmetrically partition forces between the thumb and the index finger at object lift, thus reducing object roll (Wing and Lederman, 1998). The task of Salimi et al. (2000) is similar to our predictable condition in which subjects experienced the same $\mathrm{CM}$ over several trials and therefore learned to anticipate the digit forces necessary to minimize object roll (Fig. 8). It is important to note, however, that contact points in the studies by Salimi et al. (2000) were constrained by the location of force sensors on the object. Hence, their result points out that, as noted in Introduction, subjects can modulate digit forces even when they cannot freely choose digit placement on an object.

The ability to choose contact points is an important feature that is exploited during everyday object grasping and manipulation. Specifically, subjects take into account the geometry of the object as well as planned manipulation when controlling digit motion and placement on an object (Ansuini et al., 2006; Friedman and Flash, 2006). Our results demonstrate that, when subjects can choose contact points and can anticipate object properties, they implement anticipatory force mechanisms in parallel with careful selection of digit placement. Clearly, if contact points had not been modulated to the external torque, maintaining vertical alignment of the object during lift would have forced subjects to significantly change the distribution of digit forces. Changes in contact points, however, significantly affect the moment arm of the torques generated by each digit, hence the net torque that subjects had to generate to counteract object roll. Alternatively, modulating contact points might have partially relieved the need to adopt different force sharing patterns among the digits and, possibly, the production of large forces at individual digits. The task-dependent nature of the relationship(s) between hand posture and forces, however, warrant additional investigation.

\section{References}

Ansuini C, Santello M, Massaccesi S, Castiello U (2006) Effects of end-goal on hand shaping. J Neurophysiol 95:2456-2465.

Aoki T, Niu X, Latash ML, Zatsiorsky VM (2006) Effects of friction at the digit-object interface on the digit forces in multi-finger prehension. Exp Brain Res 172:425-438.

Cohen RG, Rosenbaum DA (2004) Where grasps are made reveals how 
grasps are planned: generation and recall of motor plans. Exp Brain Res 157:486-495.

Friedman J, Flash T (2006) Selection of optimal grasps for object manipulation. Soc Neurosci Abstr 32:655.7.

Gao F, Latash ML, Zatsiorsky VM (2006) Maintaining rotational equilibrium during object manipulation: linear behavior of a highly non-linear system. Exp Brain Res 169:519-531.

Johnson KO, Phillips JR (1981) Tactile spatial resolution. I. Two-point discrimination, gap detection, grating resolution, and letter recognition. J Neurophysiol 46:1177-1192.

Johnson RA, Wichern DW (1992) Applied multivariate statistical analysis. Englewood Cliffs, NJ: Prentice Hall.

Kinoshita H, Kawai S, Ikuta K (1995) Contributions and co-ordination of individual fingers in multiple finger prehension. Ergonomics 38:1212-1230.

Lang CE, Schieber MH (2004) Reduced muscle selectivity during individuated finger movements in humans after damage to the motor cortex or corticospinal tract. J Neurophysiol 91:1722-1733.

Rearick MP, Santello M (2002) Force synergies for multifingered grasping: effect of predictability in object center of mass and handedness. Exp Brain Res 144:38-49.

Reilly KT, Hammond GR (2006) Intrinsic hand muscles and digit independence on the preferred and non-preferred hands of humans. Exp Brain Res 173:564-571.

Reilmann R, Gordon AM, Henningsen H (2001) Initiation and development of fingertip forces during whole-hand grasping. Exp Brain Res 140:443-452.

Rosenbaum DA, Vaughan J, Barnes HJ, Jorgensen MJ (1992) Time course of movement planning: selection of handgrips for object manipulation. J Exp Psychol Learn Mem Cogn 18:1058-1073.
Rosenbaum DA, van Heugten CM, Caldwell GE (1996) From cognition to biomechanics and back: the end-state comfort effect and the middle-isfaster effect. Acta Psychol (Amst) 94:59-85.

Sakitt B (1980) A spring model and equivalent neural network for arm posture control. Biol Cybern 37:227-234.

Salimi I, Hollender I, Frazier W, Gordon AM (2000) Specificity of internal representations underlying grasping. J Neurophysiol 84:2390-2397.

Santello M, Soechting JF (1998) Gradual molding of the hand to object contours. J Neurophysiol 79:1307-1320.

Santello M, Soechting JF (2000) Force synergies for multifingered grasping. Exp Brain Res 133:457-467.

Santello M, Muratori L, Gordon AM (2004) Control of multidigit grasping in Parkinson's disease: effect of object property predictability. Exp Neurol 187:517-528.

Schieber MH, Santello M (2004) Hand function: neural control and peripheral limits to performance. J Appl Physiol 96:2293-2300.

Shannon CE (1948) The mathematical theory of communication. Bell Syst Techn J 27:379-423.

Shim JK, Latash ML, Zatsiorsky VM (2005) Prehension synergies: trial-totrial variability and principle of superposition during static prehension in three dimensions. J Neurophysiol 93:3649-3658.

Short MW, Cauraugh JH (1997) Planning macroscopic aspects of manual control: end-state comfort and point-of-change effects. Acta Psychol (Amst) 96:133-147.

Wing AM, Lederman SJ (1998) Anticipating load torques produced by voluntary movements. J Exp Psychol Hum Percept Perform 24:1571-1581.

Zatsiorsky VM, Latash ML (2004) Prehension synergies. Exerc Sport Sci Rev 32:75-80. 